\title{
Clinical Efficacy of First and Second Series of Peptide Receptor Radionuclide Therapy in Patients With Neuroendocrine Neoplasm: A Cohort Study
}

Michala Danielle Zacho ( $\sim$ michalazacho@hotmail.com )

Aarhus Universitetshospital https://orcid.org/0000-0002-3008-9064

P. Iversen

Aarhus Universitetshospital

G. E. Villadsen

Aarhus Universitetshospital

S. MD Baunwall

Aarhus Universitetshospital

A.K. Arveschoug

Aarhus Universitetshospital

H. Grønbæk

Aarhus Universitetshospital

G. Dam

Aarhus Universitetshospital

\section{Research article}

Keywords: neuroendocrine, gastropancreatic tumors, bronchopulmonary tumors, peptide receptor radionuclide, first and second series

Posted Date: July 10th, 2020

DOI: https://doi.org/10.21203/rs.3.rs-36633/v1

License: (c) (i) This work is licensed under a Creative Commons Attribution 4.0 International License. Read Full License

Version of Record: A version of this preprint was published at Scandinavian Journal of Gastroenterology on January 20th, 2021. See the published version at https://doi.org/10.1080/00365521.2021.1872095. 


\section{Abstract \\ Background}

Peptide receptor radionuclide therapy (PRRT) is an established treatment for metastatic neuroendocrine neoplasms (NEN) with positive effects on progression free (PFS) and overall survival (OS), but only limited data exists for the effect of multiple series of PRRT. The aim of this study was to investigate PFS and OS in NEN patients treated with multiple series of PRRT conforming to the ENETS treatment protocol.

\section{Methods}

We included all patients with gastrointestinal (GI), pancreatic and bronchopulmonary (BP) NEN treated with PRRT from 2008 to 2018. We used Kaplan-Meier estimation to evaluate PFS and OS with subgroup analysis of primary tumor, Ki67-index, type of radioisotope and number of PRRT series.

\section{Results}

Of 150 patients, 133 patients were included with female/male 61/72, median age 70 (interquartile range 64-76) years. GI-NEN comprised $62 \%$, pancreatic $23 \%$ and BP $11 \%$. Median Ki67-index was $5 \%$. After first PRRT G1- and G2-tumors had PFS of 25 and 22 months, compared to 11 months in G3-NENs $(p<0.05)$ and PFS was longer in G1/G2 GI-NENs than BP-NEN (30 vs. 12 months, $p<0.05$ ). After retreatment with a second series of PRRT, the overall PFS (G1-G3) was 19 months, with G1- and G2-tumors having the highest PFS of 19 and 22 months, respectively. Overall, the GI and BP tumors had an OS of 54 and 51 months.

\section{Conclusions}

PRRT is an effective therapy with long-term PFS and OS, especially in G1 and G2 NENs, and with better prognosis in GI-NEN compared with BP-NENs. OS and PFS was shorter after the second series of PRRT compared with the first, however results were still encouraging.

\section{Introduction}

Neuroendocrine neoplasms (NENs) are heterogeneous malignancies arising from the diffuse endocrine system and can arise in almost all organs [1]. NEN tumors are slow growing and cure can only be achieved by surgery. Unfortunately, most NEN patients are diagnosed with metastatic disease for whom surgery is not an option [2]. This leads to the need for further options for systemic therapies [3]. Patients with inoperable and metastasized NENs usually receive treatment with somatostatin analogues (SSAs) [4] due to neuroendocrine tumors overexpression of somatostatin receptors. [5] Other treatment options 
include targeted therapy (everolimus [6], sunitinib [7]) or chemotherapy (STZ-5FU [8] and capecitabine/temozolomide [9]).

The overexpression of somatostatin receptors on the cell surface of NENs is also utilized in peptide receptor radionuclide therapy (PRRT) [10]. PRRT is an established treatment for metastatic neuroendocrine tumors that improves both progression free survival (PFS) and overall-survival (OS) [1114]. The NETTER-1 study was the first randomized prospective phase III trial comparing ${ }^{177}$ Lu-DOTATATE with high dose octreotide in patients with advanced midgut NET. The study showed superior outcome in terms of longer PFS, (28.4 vs. 8.4 months) [15]. A recent retrospective North American cohort study had similar results with a median PFS of 23.9 months and an OS from the first PRRT of 40 months [16].

The outcome of PRRT varies widely depending on tumor type, tumor biology and treatment regimen. Studies are heterogenous and only few studies have investigated the effect in NEN patients after multiple PRRT series. The Rotterdam group investigated 168 patients who received a second PRRT series and 13 patients received a third series. They found a median PFS of 14.6 months following the second treatment series and 14.2 months after the third PRRT series [17]. This need validation as little is known about the cumulative effect in several series of PRRT treatment.

The aim of this study was to evaluate the incremental PRRT treatment response in a large Danish cohort of patients with NENs following multiple series of PRRT. Patients were evaluated according to primary tumor, tumor grade and treatment type.

\section{Methods}

\section{Study design and study population}

This was a single-center, cohort study conducted at Aarhus University Hospital, ENETS Centre of Excellence. We included all patients with NENs gastrointestinal (GI), pancreatic and bronchopulmonary (BP) neuroendocrine neoplasms undergoing PRRT according to the ENETS treatment protocol [18] between 2008 and 2018. The majority of PRRT treatments were performed at the Aarhus University Hospital. However, prior to the introduction of PRRT at Aarhus University Hospital in 2008, 15 patients received their first PRRT series in Basel, Switzerland as previously reported [19]. This study included patients with NENs gastrointestinal (GI), pancreatic and bronchopulmonary (BP) NENs, who had completed at least one series of PRRT between 2008 and 2018.

Data was collected from electronic patient files documenting each patients course of treatment. We extracted data on the origin of primary tumors, proliferations-index (Ki67-index), previous therapies, dose and series of PRRTs, along with bone marrow and kidney function (including blood samples and ${ }^{51} \mathrm{Cr}$ EDTA- plasma clearance) [20].

As follow up, A 3-phase CT-scan was performed three months after the last PRRT (and subsequently continually with an interval of three months) in each series and was then discussed in multidisciplinary 
teams (MDT). The CT was combined with a Ga-68-DOTATOC PET at 6 and 12 months after the last PRRT. Status of the disease (regression, progression or stable) was based on the clinical decision at the MDTconference. Follow-up imaging and subsequent MDT-conferences were performed at intervals of 6 months after the firstrestaging. Status at the last follow-up visit was recorded.

\section{Treatment}

Prior to PRRT, all patients had undergone somatostatin receptor imaging by somatostatin receptor scintigraphy or Ga-68-DOTATOC PET/CT to assure overexpression of somatostatin receptors on tumor cells, with higher uptake than in normal liver tissue.All PRRT treatment decisions were based on a clinical decision at the MDT and included radiological progression in all cases.

The initial treatment regimen was typically planned for two or four treatments with PRRT at an interval of 8-12 weeks. This is referred to as one series of PRRT. Routine blood tests including full blood count, renal and liver function were performed and registered at week 2 and 4 following completion of each treatment series and in between series.

The treatment regimens have changed over time. From 2008 to 2015 the standard regimen was treatment with ${ }^{90}$ Y-DOTATOC, and we adopted the treatment regimens from Basel [21]. From 2015 the standard regimen was treatment with ${ }^{177}$ Lu-DOTA-peptide performed according to the EANM guidelines [18]. Combination treatment was used from 2015 in patients with G3-tumors and/or bulky tumors. The standard dose of ${ }^{177}$ Lu-DOTA-peptide was 7.4GBq. In 2008 to 2015 the standard dose of ${ }^{90}$ Y-DOTATOC was $3.7 \mathrm{GBq} / \mathrm{m} 2$ body surface (a maximum doses of $7.4 \mathrm{GBq} /$ doses), distributed over two treatments with an interval of 8-10 weeks. In 2013 this was changed to four treatments (with an interval of 8-10 weeks) with a standard dose at $1.85 \mathrm{GBq} / \mathrm{m} 2$ body surface $[12,22]$. The decision to re-treat patients with a second or third cycle was primarily based on combination of the duration of response, toxicity and degree of uptake on SSTR-PET. Patients who developed progression within 12 months were excluded from retreatment.

Kidney protection was planned according to baseline estimated glomerular filtration rate (eGFR) and ${ }^{51} \mathrm{Cr}$ EDTA-plasma clearance (Cr-GFR). To reduce the radiation dose to the kidneys and thereby reducing the nephrotoxic effects of the treatment, amino acid solutions were administered before, and continued after injection to inhibit tubular reabsorption. From 2008 to 2015 all patients received nephroprotection with a commercial mixture (Vamin-18, Fresenius Kabi AG, Bad Homburg, Germany). The mixture contains a combination of various AA, the most abundant being L-alanine $(16,0 \mathrm{~g} / 1000 \mathrm{ml})$, L-arginine $(11,3 \mathrm{~g} / 1000$ $\mathrm{ml})$ and L-lysine $(9,0 \mathrm{~g} / 1000 \mathrm{ml})$. From 2015 all patients received a solution containing arginine and lysine Rolleman-solution [23] (1,000 $\mathrm{ml}$ of $0.9 \% \mathrm{NaCl}$ solution containing 2,5\% arginine and 2,5\% lysine), according to the so-called Rotterdam protocol [18]. In patients with an e-GFR $>50 \mathrm{ml} / \mathrm{min}$ a solution containing a $50-\mathrm{g}$ cocktail of lysine and arginine ( $25 \mathrm{~g}$ of lysine and $25 \mathrm{~g}$ of arginine) was diluted in $2 \mathrm{I}$ of normal saline and infused over $4 \mathrm{~h}$, starting 30-60 min before PRRT. If the kidney function was decreased (e-GFR $<50 \mathrm{ml} / \mathrm{min}$ and $>40 \mathrm{ml} / \mathrm{min}$ ) 2 liters of lysine $(2,5 \%)$ and arginine $(2,5 \%)$ mixture with 1 liter of saline was infused over $12 \mathrm{~h}$, starting $60 \mathrm{~min}$ before PRRT and continuing over 12 hours with 
$170 \mathrm{ml} /$ hour. An extra bolus of $1 / 2$ liter of the mixture 24 hours after PRRT. In patients with e-GFR 30-40 $\mathrm{ml} / \mathrm{min} 3$ liters of lysine (2,5\%) and arginine (2,5\%) mixture with 1-2 liter of saline, was infused over $24 \mathrm{~h}$, starting 60 min before PRRT and continuing over 24 hours with $125 \mathrm{ml}$. per hour. An extra bolus of 1/2 liter of the mixture is given 48 hours after PRRT $[20,24]$.

\section{Statistical analyses}

Patient data were captured and managed in an electronic REDCap database hosted at Aarhus University[25]. The baseline characteristics of the study population were stratified with regard to primary tumor site. Continuous data are presented as medians with interquartile ranges (IQR) $25^{\text {th }}-75^{\text {th }}$ percentiles, and categorical data as proportions with percentages. The PFS was calculated as months from the date of the first treatment of PRRT to the date of progression or death of any course. OS was calculated from the first day of treatment until the day of death of any course.Kaplan-Meier estimates were used to determine PFS and OS, by assessing the time between the date of the first PRRT to date of progression or death of any cause (or to the date of censoring for patients known to be alive). Subgroup analyses were performed according to the primary tumor site, Ki67-index, previous surgery, type of PRRT (isotope) and number of PRRT series. Patients were censored at the last follow-up if they were without progression and at start of analysis $\left(27^{\text {th }}\right.$ august 2019$)$ if they were still alive. The subgroups were compared using an uni-stratified log-rank test. A value of $p<0.05$ was considered statistically significant. Hazard ratios were estimated by uni-variate proportional hazards Cox-model. Statistical software (Prism, version 8.0) was used to analyze the data.

\section{Ethical statement}

The study was approved by the local ethics committee of Central Region Midt-Jutland (656236).

\section{Results}

\section{Patients}

From 2008-2018 a total of 150 patients, 61 (46\%) female and 72 (54\%) male, were treated at the department of Hepatology and Gastroenterology Aarhus (15 were treated in Basel before treatment in Aarhus [19]). Their median age was 70 years (IQR 64-76). Ten patients (6.6\%) with paragangliomas and meningioma were excluded. Seven patients $4.6 \%$ ) were excluded as they had either not completed a full series of PRRT or were awaiting follow-up evaluation at the time of analysis. Six patients did not have a follow-up visit before time of death. We included 133 (89\%) patients for the final analysis.

Information on the Ki-67 index of the primary tumor or metastases was available for 123 patients (92\%). The majority of the patients (63\%) had G2 NET with median Ki-67 index 5\% (range 3-15\%). Twenty-four patients (20\%) had G1 tumors (defined as Ki-67 index $\leq 2 \%$ ) and 21 (17\%) had a G3 tumor (Ki-67 index $>20$ ). Baseline characteristics including location of primary tumor, disease stage and previous medical treatment and interventions are shown in Table 1. 
In the first series, $66(50 \%)$ patients were treated with ${ }^{90} \mathrm{Y}$-DOTATOC, $60(45 \%)$ were treated with ${ }^{177} \mathrm{Lu}$ DOTA-peptide and 7 (5\%) with the combination of the two.

A second PRRT series was given to $36(27 \%)$. Among these patients, $8(23 \%)$ were treated with ${ }^{90} \mathrm{Y}$ DOTATOC, 25 (69\%) with ${ }^{177}$ Lu-DOTA-peptid and 3 (8\%) with combination.

A third series of PRRT was offered to 8 patients (6\%), where $2(25 \%)$ received with ${ }^{90} \mathrm{Y}$-DOTATOC and 6 (75\%) with ${ }^{177}$ Lu-DOTA-peptid. (Table 2)

\section{Progression free survival}

The final number enrolled into the survival analysis was 133 patients. Six (5\%) patients died before follow-up and 7 patients (5\%) did not have a follow up visit after their second or third series of PRRT.

\section{First series}

A CT was performed 3 months after the last PRRT cycle in a treatment series. In all patients with G1 and $\mathrm{G} 2$ tumors, regardless of primary tumor and the radiopharmaceutical administered, we observed progression in 83 patients $(62 \%)$ and disease regression in 50 patients (38\%) after the first series of PRRT. The overall median PFS was 25 (11-41) months. In figure 1A we show the PFS according to primary tumor site (GI, pancreatic and BP) in G1 and G2 NEN patients. PFS was 30 (12-41), 19 (9-53) and 12 (922) months, respectively, and with a significant difference between $\mathrm{GI}$ and BP NETs ( $p$-value<0.05), and with an associated odds ratio of 0.33 (0.13-0.84).

PFS was compared according to tumor grade as shown in Figure 1B. The G1- and G2-tumors had a PFS of 25 (11-36) and 22 (10-41) months, while the G3-tumor had a PFS of 11 (5-29) months (NS).

There was no significant difference in PFS between patients treated with ${ }^{177} \mathrm{Lu}$ or ${ }^{90} \mathrm{Y}$ in the first series of PRRT with median PFS of 28 (10-35) months and 22 (10-40) months, respectively (NS).

Time from last cycle in the first series to first cycle in second series .

In all patients (with completion of at least two series of PRRT) the median time from the last treatment in the first series to the first treatment in the second series was 21 months. The time for the Gl-tumors $(n=21)$ was 20 months, while it was 30 months for pancreatic tumors $(n=8)$ and 29 months for pulmonary tumors $(n=4)(N S)$. The time for the $G 1$ tumors $(n=8)$ was 19 months, while it was 33 months for $\mathrm{G} 2$ tumors $(n=22)$ and 21 months for $\mathrm{G} 3$ tumors $(n=3)(N S)$.

\section{Second series}

After the second series of PRRT, progression was observed in 23 patients (64\%) with a median PFS of 19 (10-32) months, including all patients with G1 and G2 tumors. Only 3 patients with G3 tumors received a 
second series of PRRT.

When G1- and G2-tumors were divided according to primary tumor we found a higher median PFS for GINET of 19 (9-26) months, compared to 33 (11-36) months for pancreas and 26 (4-19) months for BP-NET (Figure 2A) $(p<0.05)$.

G1- and G2-tumors showed a PFS of 19 (6-not reached) and 22 (10-33) months, respectively. The G3 tumors had a PFS of 4 (1-16) months. (Figure 2B) (NS)

Patients treated with ${ }^{177} \mathrm{Lu}$ in the second series of PRRT had a PFS of 16 (9-33) months, while patients treated with ${ }^{90} \mathrm{Y}$ had a PFS of 22 (9-29) months. (NS)

\section{PFS by number of PRRT series}

The median PFS after the first $(n=133)$ and second series $(n=32)$, was 21 (10-41) vs. 17 (10-27) months, while PFS was 12 (8-15) months after the third series $(n=8)$ with a significant difference in PFS after the first and third series $(p<0.05)$.

\section{Overall survival}

65 patients (49\%) died during follow up. The median OS for the whole cohort was 51 (21-119) months. The median OS for the G1-tumors was not reached whereas the G2 and G3 tumors had an OS of 53 months (21-128) and 31 (10-87) months, respectively (Figure 3A) (NS). In addition, we divided all G1 and G2 tumors into three subgroups according to primary tumor site. The Gl-tumors had an OS of 54 (31-123) months, while pancreatic- and BP tumors had an OS of 51 (15-72) and 40 (10- 75 percentile not reached) months, respectively (Figure 3B (NS).

\section{Bone marrow- and kidney function}

Hemoglobin $(\mathrm{HgB})$, leukocytes and platelets were used to evaluate the side effects of PRRT on bone marrow function, while ${ }^{51} \mathrm{Cr}$-EDTA-plasma clearance measurements were used assess kidney function. Cr-51-GFR plasma clearance was measured before, 3, 6 and 12 months after treatment with ${ }^{177}$ Lu-DOTApeptide, and with ${ }^{90} \mathrm{Y}$-DOTATOC it was measured before, 3,6,12 and occasionally 18 and 24 months after PRRT. In between treatments kidney function was monitored with estimated Glomerular filtration rate also called estimated GFR (eGFR). Within our institution the formula to estimate the eGFR is the Modification of Diet in Renal Disease (MDRD) formula [20].

Baseline laboratory assessments were compared with data obtained at the end of treatment (Table 3). We found that in those treated with ${ }^{90} \mathrm{Y}$ the Cr-GFR was reduced by an average of $4 \%, 7 \%$ and $12 \%$, when comparing Cr-GFR before and after first, second and third series of PRRT. In patients treated with ${ }^{177} \mathrm{LU}$ the Cr-GFR was reduced by $3 \%$ and $7 \%$ after first and second series, whereas an increase of $3 \%$ was observed after the third series. In patients treated with the combination, we found a decrease in Cr-GFR of $9 \%$ and $4 \%$ after first and second series of PRRT. 
Common Toxicity Criteria Scale version 3.0 were used for evaluation [26] and adverse events were accounted severe according to grades III and IV. We found temporary grade III and IV anemia in $4 \%$ ( 3 cases of grade III: Hgb 4.0 to $<4.9 \mathrm{mmol} / \mathrm{l} ; 2$ case of grade IV: $\mathrm{Hgb}<4.0 \mathrm{mmol} / \mathrm{l})$. Three patients treated with $\mathrm{Lu}^{177}$ and 3 patients treated with $\mathrm{Y}^{90}$ (Table 4). Leucopenia grade III was found in one patient (total white blood cells $\geq 1.0$ to $<2.0 \times 10^{9} /$ I), treated with $\mathrm{Lu}^{177}$. No temporary grade III or IV thrombocytopenia was observed.

Due to the retrospective nature of the evaluation, valid numbers on acute side effects could not be provided

\section{Discussion}

This large cohort study confirms an excellent median progression free and overall survival following the first series of PRRT in patients with GEP and BP NEN. Interestingly, unlike previous studies, this study is the first study demonstrating a favorable response also after a second series of PRRT with a progression free survival of 19 months across all primary tumors. An explanation may be higher proportion of patients with low grade NEN in the group receiving a second series of PRRT compared with other studies. This underline that a second series of PRRT is feasible in selected patients showing progression after their first PRRT series.

Our results are comparable to data from the randomized NETTER-1 study, which only included well differentiated, metastatic GEP-NETs treated with ${ }^{177}$ Lu-DOTATATE [15]. They reported a PFS of 28.4 months compared to our 25 months; however, we also included BP-NETs, who have a shorter PFS $(p<0.05)$. Results also corresponds with the data from another Danish cohort of G1- and G2-GEP NET patients treated in Basel reporting a PFS of 29 months [19]. Recently, Sharma et al reported outcomes in a North American cohort with metastatic well-differentiated GEP NETs with a PFS of 24 months. In their cohort both the PFS and the OS was higher in patients with pancreatic NETs compared to GI-NETs [16]. This was also the case in a retrospective study of pancreatic NET G1/2 tumors, which demonstrated a PFS of 34 months and an OS of 53 months [27]. Both these studies are in contrast to our findings where both the PFS and the OS was higher in patients with Gl-tumors (NS). This may be explained by a higher Ki67-index in the pancreas group compared to the GI group. In accordance with previous studies, we also observed a significant difference between the PFS of GI and BP NETs [10, 11]. A recent review summarized several results from previous studies where PFS varied from 17 to 29 months and median OS from 22 to 37 months with differences according to primary tumor and Ki-index [28]. Overall, the PRRT results seem to be rather consistent across the literature.

The median OS in our cohort across all groups was 51 months. There was no significant difference between the OS across the three subgroups, but a strong trend indicated a better survival in G1 and G2 tumors compared with the G3 tumors. The GI-NETs had an OS of 54 months while the OS was 51 months for pancreatic- and 40 months for BP tumors (NS). These results seem to be slightly better than the ones demonstrated by Sharma et al with an OS of 40 months in their GEP NET G1/2 cohort [16]. 
Only a few studies have included G3 tumors. Ezziddin et al. demonstrated that a Ki-67 index of $<20 \%$ was predictive of a good response following PRRT while a Ki-67 $>20 \%$ was associated with progression within 3 months after PRRT treatment [29]. Aalbersberg et al. also showed that higher Ki-67-values was a negative predictor for both PFS and OS [30]. Further, some studies [31, 32] showed an inverse association between tumor grade and tumor uptake on somatostatin receptor imaging. However, patients are scanned to assure overexpression of somatostatin receptors before starting PRRT. That said, our patients with Ki-67 $>20 \%$ had a fairly response of PRRT with a PFS of 12 months after the first series of PRRT.

The possible benefit in high-grade GEP NEC has until recently been debated. A recent large retrospective cohort study of 149 GEP NEC G3 patients demonstrated promising results [33]. Their results were better in the subgroup with Ki-67 between $21-54 \%$ vs Ki-67 $\geq 55 \%(P<0.001)$. Overall, the total cohort had a median PFS of 14 months and OS of 29 months [33]. These results correspond well with our NEC cohort including BP NECs as we demonstrated a PFS of 11 months and an OS of 31 months. Hence, our results support the use of PRRT also in NEC patients.

Previous studies investigating PFS in NEN patients after the second PRRT series are limited. Recently, results from the large Rotterdam cohort reported a PFS after a second series of PRRT of 15 months, including GEP- and bronchial G1/2 NETs [17]. They also compared PFS according to primary tumor site (pancreatic $(n=53)$, bronchial $(n=8)$ and midgut $(n=54)$ ), where PFS was 14,8 and 5 months, respectively. Additionally, Vaughan et al reported a median PFS of 18 months [34] after retreatment, whereas Severi et al demonstrated a PFS of 22 months [35] compared to the 12 months in the study by Yordanova [36]. In contrast a previous smaller study including 35 mainly GI and BP G2 NET showed the shortest PFS of six months. This is surprising as progression in that study was defined according to RECIST (response evaluation criteria in solid tumors) which would statistically lead to a longer PFS compared to the studies using clinical progression [37].

Our cohort demonstrated an overall PFS of 19 months for GI-NET ( $n=19), 33$ months for pancreatic $(n=3)$ and 26 months for BP $(n=5)$ NET after the second PRRT series. The distribution of tumor grade in our study was similar compared to the Rotterdam study [17]. Our analysis is based on few patients and firm conclusions should be drawn with caution. However, despite the lower PFS compared to the first series, we believe that a second series of PRRT makes sense as it results in a fairly long PFS and OS. The very fast progression in G3 tumors, which were given a second series, was based on very few patients.

Only one trial investigated PFS in patients receiving a third series of PRRT and reported a median PFS of 15 months [17]. They included 13 patients with GI- $(n=4)$, pancreatic- $(n=4), B P(n=2)$ and unknown primary $(n=3)$ NETs. These results correspond with ours with a PFS after a third series of PRRT of 12 months, including $\mathrm{GI}(\mathrm{n}=5)$, pancreatic $(n=1), B P(n=1)$ and unknown $(n=1)$ NETs. The median Ki67-index was $5 \%$. Data are limited but we believe that a third series may be favorable in selected patients.

Patients in this study were treated either with ${ }^{177} \mathrm{Lu},{ }^{90} \mathrm{Y}$ or the combination. Patients treated with ${ }^{177} \mathrm{Lu}$ in the first PRRT had a median PFS of 28 months, while patients treated with ${ }^{90} \mathrm{Y}$ had a PFS of 21 months 
(NS). ${ }^{90} \mathrm{Y}$ has larger beta energy and better tissue penetration than ${ }^{177} \mathrm{Lu}$, and in combination with DOTATOC it was used the first seven years. The standard regimes have changed over time and after 2015 we used ${ }^{177}$ Lu-DOTApeptid as standard treatment, and in 2018 only one patient was treated with ${ }^{90} \mathrm{Y}$ DOTATOC. However, the study was retrospective and the role of ${ }^{90} \mathrm{Y}$-DOTATOC has changed, and the ${ }^{90} \mathrm{Y}$ labelled peptide is by now preferably used in patients with large bulky tumors, which may explain the tendency towards a less favorable result.

Due to the larger beta energy and the better tissue penetration, we would expect that ${ }^{90} \mathrm{Y}$ would have a larger effect on the kidney and bone marrow function than ${ }^{177} \mathrm{Lu}$. We did not find a significance difference between the side effects of the two treatments. Some missing data might explain the lack of difference between the two treatments; however, the missing data were evenly distributed among the patients, who received treatment with ${ }^{90} \mathrm{Y}$ and ${ }^{177} \mathrm{Lu}$. In accordance with the NETTER-1 trial we observed only mild toxicity in the majority of the patients, and we did not observe any difference in the decrease in kidney function when comparing ${ }^{90} \mathrm{Y}$ and ${ }^{177} \mathrm{Lu}$.

The major strength of the present study is the high number of well-characterized NEN patients treated at a highly specialized ENETs center of excellence. Follow-up data was almost complete due the standarsized treatment course. We included patients starting PRRT in 2008, allowing a long follow-up period, and we used the same Case Report Form (CRF) for all patients.

The study has several limitations. Data from the first treatments completed in Basel and Copenhagen were not as complete as the treatments completed in Aarhus. This, in addition to the patient heterogeneity with regard to primary tumor and $\mathrm{Ki}-67$ index, may have influenced the outcomes. In this cohort, the standard PRRT treatment has changed over the 10-year period since new treatments and new clinical guidelines develop over time. In 2013, the standard PRRT series was changed from two to four ${ }^{90}$ Yttrium treatments with 8-12 weeks intervals, and in late 2015 we implemented the use of ${ }^{77}$ Luteium as a first-line treatment. This inconsistency introduces an overtime bias and firm conclusion must be taken with regard to improvements over time. Our response evaluation was not standardized as it was based on the clinical decision at a dedicated NET MDT-conference, rather than based on the RECIST. Progression, however, always included radiological progression and was probably decided at an earlier stage compared with other studies where progression was based on RECIST. Finally, the comparison with other studies should be done with caution due to different treatment protocols, differences in patient populations and disease stages, and with differences in inclusions- and exclusions criteria and follow-up protocols.

\section{Conclusion}

In this large cohort study on effects of a first and second series of PRRT treatment at Aarhus University Hospital, we demonstrated an excellent progression free survival with a median of 25 months and an overall survival of 51 months after the first series of PRRT in patients with GEP and BP NET. Tumors 
originating from the Gl-tract had a better response than BP tumors, and G1- and G2-tumours responded better than G3. Interestingly, we observed a favorable PFS of 19 months after a second series of PRRT independent on tumor grade. Our findings thus suggest that a second series of PRRT may be favorable in selected patients progressing after their first PRRT series.

\section{Abbreviations}

NEN Neuroendocrine neoplasm

NET Neuroendocrine tumor

NEC Neuroendocrine carcinoma

PRRT Peptide receptor radionuclide therapy

GI Gastrointestinal

BP Bronchopulmonary

GEP Gastroenteropancreatic

G1/2/3 Grade 1/2/3

ENETS European Neuroendocrine Tumor Society

RECIST Response evaluation criteria in solid tumors

CrGFR Glomerular filtration rate measured by Cr-EDTA plasma clearance

OS Overall survival

PFS Progression free survival

177-Lu 177-Lutetium

99-Y 99-Yttrium

NS Non-significant

\section{Declarations}

Ethics approval and consent to participate

The study was approved by the local ethics committee of Central Region Midt-Jutland and according to national regulations the need for consent was waived (656236).

11109099

Consent for publication

Not applicable.

Availability of data and materials

The datasets used and/or analyzed during the current study are available from the corresponding author on reasonable request. 
H. Grønbæk has received research grants from Abbvie, Intercept and NOVO Nordisk Foundation, and is on advisory board at Ipsen and speaker for Norgine. The other authors declare that they have no competing interests.

\section{Funding}

None.

\section{Author Contributions}

H. Grønbæk, G. Dam and M.D. Zacho conceived the study.

M.D. Zacho extracted the data and performed data analysis in collaboration with G. Dam, H. Grønbæk and S. MD Baunwall.

P. Iversen, G. E. Villadsen and A. K. Arveschoug evaluated treatment responses.

All authors participated in drafting the manuscript and all authors approved the final version.

\section{Acknowledgements:}

Not applicable.

\section{References}

1. Lawrence B, Gustafsson BI, Chan A, Svejda B, Kidd M, Modlin IM. The epidemiology of gastroenteropancreatic neuroendocrine tumors. Endocrinol Metab Clin North Am. 2011;40(1):1-18, vii.

2. Oberg K. Biotherapies for GEP-NETs. Best Pract Res Clin Gastroenterol. 2012;26(6):833-41.

3. Uri I, Avniel-Polak S, Gross DJ, Grozinsky-Glasberg S. Update in the Therapy of Advanced Neuroendocrine Tumors. Curr Treat Options Oncol. 2017;18(12):72.

4. Reubi JC, Waser B. Concomitant expression of several peptide receptors in neuroendocrine tumours: molecular basis for in vivo multireceptor tumour targeting. Eur J Nucl Med Mol Imaging. 2003;30(5):78193.

5. Oberg KE, Reubi JC, Kwekkeboom DJ, Krenning EP. Role of somatostatins in gastroenteropancreatic neuroendocrine tumor development and therapy. Gastroenterology. 2010;139(3):742-53, 53.e1.

6. Chan J, Kulke M. Targeting the mTOR signaling pathway in neuroendocrine tumors. Curr Treat Options Oncol. 2014;15(3):365-79. 
7. Faivre S, Niccoli P, Castellano D, Valle JW, Hammel P, Raoul JL, et al. Sunitinib in pancreatic neuroendocrine tumors: updated progression-free survival and final overall survival from a phase III randomized study. Ann Oncol. 2017;28(2):339-43.

8. Naidoo J, O'Toole D, Kennedy MJ, Reynolds JV, O'Connor M, O'Byrne K. A single institution experience of streptozocin/fluorouracil combination chemotherapy: a case series. Ir J Med Sci. 2012;181(2):211-4.

9. Ramirez RA, Beyer DT, Chauhan A, Boudreaux JP, Wang YZ, Woltering EA. The Role of Capecitabine/Temozolomide in Metastatic Neuroendocrine Tumors. Oncologist. 2016;21(6):671-5.

10. Imhof A, Brunner P, Marincek N, Briel M, Schindler C, Rasch $\mathrm{H}$, et al. Response, survival, and long-term toxicity after therapy with the radiolabeled somatostatin analogue [90Y-DOTA]-TOC in metastasized neuroendocrine cancers. J Clin Oncol. 2011;29(17):2416-23.

11. Brabander T, van der Zwan WA, Teunissen JJM, Kam BLR, Feelders RA, de Herder WW, et al. LongTerm Efficacy, Survival, and Safety of [(177)Lu-DOTA(0),Tyr(3)]octreotate in Patients with Gastroenteropancreatic and Bronchial Neuroendocrine Tumors. Clin Cancer Res. 2017;23(16):4617-24.

12. Bodei L, Cremonesi M, Grana C, Rocca P, Bartolomei M, Chinol M, et al. Receptor radionuclide therapy with 90Y-[DOTA]0-Tyr3-octreotide (90Y-DOTATOC) in neuroendocrine tumours. Eur J Nucl Med Mol Imaging. 2004;31(7):1038-46.

13. Severi S, Grassi I, Nicolini S, Sansovini M, Bongiovanni A, Paganelli G. Peptide receptor radionuclide therapy in the management of gastrointestinal neuroendocrine tumors: efficacy profile, safety, and quality of life. Onco Targets Ther. 2017;10:551-7.

14. Kwekkeboom DJ, de Herder WW, Kam BL, van Eijck CH, van Essen M, Kooij PP, et al. Treatment with the radiolabeled somatostatin analog [177 Lu-DOTA 0,Tyr3]octreotate: toxicity, efficacy, and survival. J Clin Oncol. 2008;26(13):2124-30.

15. Strosberg J, El-Haddad G, Wolin E, Hendifar A, Yao J, Chasen B, et al. Phase 3 Trial of (177)LuDotatate for Midgut Neuroendocrine Tumors. N Engl J Med. 2017;376(2):125-35.

16. Sharma N, Naraev BG, Engelman EG, Zimmerman MB, Bushnell DL, Jr., O'Dorisio TM, et al. Peptide Receptor Radionuclide Therapy Outcomes in a North American Cohort With Metastatic Well-Differentiated Neuroendocrine Tumors. Pancreas. 2017;46(2):151-6.

17. van der Zwan WA, Brabander T, Kam BLR, Teunissen JJM, Feelders RA, Hofland J, et al. Salvage peptide receptor radionuclide therapy with [(177)Lu-DOTA,Tyr(3)]octreotate in patients with bronchial and gastroenteropancreatic neuroendocrine tumours. Eur J Nucl Med Mol Imaging. 2019;46(3):704-17.

18. Bodei L, Mueller-Brand J, Baum RP, Pavel ME, Horsch D, O'Dorisio MS, et al. The joint IAEA, EANM, and SNMMI practical guidance on peptide receptor radionuclide therapy (PRRNT) in neuroendocrine tumours. Eur J Nucl Med Mol Imaging. 2013;40(5):800-16.

Page 13/19 
19. Pfeifer AK, Gregersen T, Gronbaek H, Hansen CP, Muller-Brand J, Herskind Bruun K, et al. Peptide receptor radionuclide therapy with Y-DOTATOC and (177)Lu-DOTATOC in advanced neuroendocrine tumors: results from a Danish cohort treated in Switzerland. Neuroendocrinology. 2011;93(3):189-96.

20. Arveschoug AK, Kramer SM, Iversen P, Frokiaer J, Gronbaek H. Monitoring Kidney Function in Neuroendocrine Tumor Patients Treated with (90)Y-DOTATOC: Associations with Risk Factors. Curr Radiopharm. 2015;8(1):49-55.

21. Nicolas G, Giovacchini G, Müller-Brand J, Forrer F. Targeted radiotherapy with radiolabeled somatostatin analogs. Endocrinol Metab Clin North Am. 2011;40(1):187-204, ix-x.

22. Valkema R, Pauwels SA, Kvols LK, Kwekkeboom DJ, Jamar F, de Jong M, et al. Long-term follow-up of renal function after peptide receptor radiation therapy with (90)Y-DOTA(0),Tyr(3)-octreotide and (177)LuDOTA(0), Tyr(3)-octreotate. J Nucl Med. 2005;46 Suppl 1:83s-91s.

23. Rolleman EJ, Valkema R, de Jong M, Kooij PP, Krenning EP. Safe and effective inhibition of renal uptake of radiolabelled octreotide by a combination of lysine and arginine. Eur $\mathrm{J}$ Nucl Med Mol Imaging. 2003;30(1):9-15.

24. Bodei L, Cremonesi M, Zoboli S, Grana C, Bartolomei M, Rocca P, et al. Receptor-mediated radionuclide therapy with 90Y-DOTATOC in association with amino acid infusion: a phase I study. Eur J Nucl Med Mol Imaging. 2003;30(2):207-16.

25. Harris PA, Taylor R, Thielke R, Payne J, Gonzalez N, Conde JG. Research electronic data capture (REDCap)-a metadata-driven methodology and workflow process for providing translational research informatics support. J Biomed Inform. 2009;42(2):377-81.

26. Common Terminology Criteria for Adverse Events v3.0 (CTCAE) August 9, 2006 [Available from: https://ctep.cancer.gov/protocolDevelopment/electronic_applications/docs/ctcaev3.pdf.

27. Ezziddin S, Khalaf F, Vanezi M, Haslerud T, Mayer K, Al Zreiqat A, et al. Outcome of peptide receptor radionuclide therapy with $177 \mathrm{Lu}$-octreotate in advanced grade $1 / 2$ pancreatic neuroendocrine tumours. Eur J Nucl Med Mol Imaging. 2014;41(5):925-33.

28. Hirmas N, Jadaan R, Al-lbraheem A. Peptide Receptor Radionuclide Therapy and the Treatment of Gastroentero-pancreatic Neuroendocrine Tumors: Current Findings and Future Perspectives. Nucl Med Mol Imaging. 2018;52(3):190-9.

29. Ezziddin S, Attassi M, Yong-Hing CJ, Ahmadzadehfar H, Willinek W, Grunwald F, et al. Predictors of long-term outcome in patients with well-differentiated gastroenteropancreatic neuroendocrine tumors after peptide receptor radionuclide therapy with 177Lu-octreotate. J Nucl Med. 2014;55(2):183-90.

30. Aalbersberg EA, Huizing DM, Walraven I, de Wit-van der Veen BJ, Kulkarni HR, Singh A, et al.

Parameters to predict progression free and overall survival after peptide receptor radionuclide therapy: a 
multivariate analysis in 782 patients. J Nucl Med. 2019.

31. Ezziddin S, Logvinski T, Yong-Hing C, Ahmadzadehfar H, Fischer HP, Palmedo H, et al. Factors predicting tracer uptake in somatostatin receptor and MIBG scintigraphy of metastatic gastroenteropancreatic neuroendocrine tumors. J Nucl Med. 2006;47(2):223-33.

32. Kayani I, Bomanji JB, Groves A, Conway G, Gacinovic S, Win T, et al. Functional imaging of neuroendocrine tumors with combined PET/CT using 68Ga-DOTATATE (DOTA-DPhe1,Tyr3-octreotate) and 18F-FDG. Cancer. 2008;112(11):2447-55.

33. Carlsen EA, Fazio N, Granberg D, Grozinsky-Glasberg S, Ahmadzadehfar H, Grana CM, et al. Peptide receptor radionuclide therapy in gastroenteropancreatic NEN G3: a multicenter cohort study. Endocr Relat Cancer. 2019;26(2):227-39.

34. Vaughan E, Machta J, Walker M, Toumpanakis C, Caplin M, Navalkissoor S. Retreatment with peptide receptor radionuclide therapy in patients with progressing neuroendocrine tumours: efficacy and prognostic factors for response. Br J Radiol. 2018;91(1091):20180041.

35. Severi S, Sansovini M, Ianniello A, Bodei L, Nicolini S, Ibrahim T, et al. Feasibility and utility of retreatment with (177)Lu-DOTATATE in GEP-NENs relapsed after treatment with (90)Y-DOTATOC. Eur J Nucl Med Mol Imaging. 2015;42(13):1955-63.

36. Yordanova A, Mayer K, Brossart P, Gonzalez-Carmona MA, Strassburg CP, Essler M, et al. Safety of multiple repeated cycles of (177)Lu-octreotate in patients with recurrent neuroendocrine tumour. Eur $\mathrm{J}$ Nucl Med Mol Imaging. 2017;44(7):1207-14.

37. Rudisile S, Gosewisch A, Wenter V, Unterrainer M, Boning G, Gildehaus FJ, et al. Salvage PRRT with (177)Lu-DOTA-octreotate in extensively pretreated patients with metastatic neuroendocrine tumor (NET): dosimetry, toxicity, efficacy, and survival. BMC Cancer. 2019;19(1):788.

\section{Tables}

Table 1. Patient characteristics at baseline

Abbreviations: IQR = interquartile range. Gl: gastrointestinal (includes small intestines and colon). RFA: radiofrequency ablation. TAE: transarteriel embolization. Ki-67 index was available for 123 (92\%). Chemotherapy includes Sunitinib, STZ/5FU, Temozolomid/Capecitabin, Carboplatin/Etoposid. 


\begin{tabular}{|c|c|c|c|c|c|}
\hline Primary tumor & $\begin{array}{l}\text { All sites } \\
(\mathbf{n}=\mathbf{1 3 3})\end{array}$ & $\begin{array}{l}\text { Pancreas } \\
(\mathrm{n}=\mathbf{3 1})\end{array}$ & $\begin{array}{l}\text { GI } \\
(n=82)\end{array}$ & $\begin{array}{l}\text { Pulmonary } \\
(\mathrm{n}=14)\end{array}$ & $\begin{array}{l}\text { Other sites } \\
(\mathbf{n}=6)\end{array}$ \\
\hline Age, median (IQR) & $70(64-76)$ & $67(59-75)$ & $71(66-76)$ & $70(63-75)$ & $66(62-71)$ \\
\hline $\begin{array}{c}\text { Gender, n (\%) } \\
\text { Female } \\
\text { Male }\end{array}$ & $\begin{array}{l}61(46) \\
72(54)\end{array}$ & $\begin{array}{l}13(42) \\
18(58)\end{array}$ & $\begin{array}{l}41(50) \\
41(50)\end{array}$ & $\begin{array}{l}4(29) \\
10(71)\end{array}$ & $\begin{array}{l}3(50) \\
3(50)\end{array}$ \\
\hline $\begin{array}{l}\text { Organ metastases, } \mathbf{n}(\%) \\
\text { Local } \\
\text { Liver } \\
\text { Bone } \\
\text { Other }\end{array}$ & $\begin{array}{l}89(67) \\
115(87) \\
40(30) \\
14(11)\end{array}$ & $\begin{array}{l}16(52) \\
28(90) \\
8(26) \\
3(10)\end{array}$ & $\begin{array}{l}61(74) \\
72(90) \\
18(22) \\
8(10)\end{array}$ & $\begin{array}{l}9(64) \\
11(79) \\
12(86) \\
1(7)\end{array}$ & $\begin{array}{l}3(40) \\
4(67) \\
2(33) \\
2(33)\end{array}$ \\
\hline $\begin{array}{l}\text { Ki67-index, median (IQR) } \\
\quad \leq 2 \\
>2 \leq 20 \\
>20\end{array}$ & $\begin{array}{l}5(3-15) \\
24(20) \\
78(63) \\
21(17)\end{array}$ & $\begin{array}{l}10(5-25) \\
1(3) \\
21(68) \\
9(29)\end{array}$ & $\begin{array}{l}5(2-10) \\
23(28) \\
45(55) \\
7(9)\end{array}$ & $\begin{array}{l}10(5-15) \\
0 \\
11(85) \\
2(15)\end{array}$ & $\begin{array}{l}40(23-40) \\
0 \\
1(17) \\
3(50)\end{array}$ \\
\hline $\begin{array}{l}\text { Previous treatment, n (\%) } \\
\text { Surgery } \\
\text { Somatostatinanalogues } \\
\text { Interferones } \\
\text { Chemotherapy } \\
\text { RFA/TAE }\end{array}$ & $\begin{array}{l}52(39) \\
113(85) \\
42(37) \\
67(51) \\
11(8)\end{array}$ & $\begin{array}{l}5(16) \\
21(68) \\
7(23) \\
28(90) \\
3(10)\end{array}$ & $\begin{array}{l}43(52) \\
78(97) \\
27(31) \\
20(24) \\
7(9)\end{array}$ & $\begin{array}{l}6(43) \\
10(77) \\
6(46) \\
8(62) \\
1(8)\end{array}$ & $\begin{array}{l}1(17) \\
4(67) \\
2(33) \\
5(83) \\
0(0)\end{array}$ \\
\hline
\end{tabular}

Table 2. Peptid Receptor Radionuclide Therapy

Number of patients who received one, two, three or four cycle in the 1st, 2nd or 3rd series of PRRT *() percentage of the patients who received the given isotype

\begin{tabular}{|c|c|c|c|c|c|c|c|c|c|c|}
\hline & \multicolumn{5}{|c|}{${ }^{90} \mathbf{Y}$-DOTATOC } & \multicolumn{5}{|c|}{${ }^{177}$ Lu-DOTA-peptid } \\
\hline & \multirow[t]{2}{*}{$\begin{array}{c}\text { Administered } \\
\text { activity }(\mathrm{GBq})\end{array}$} & \multicolumn{4}{|c|}{ No. of cycles* } & \multirow[t]{2}{*}{$\begin{array}{l}\text { Administered } \\
\text { activity }(\mathrm{GBq})\end{array}$} & \multicolumn{4}{|c|}{ No. of cycles* } \\
\hline & & I & II & III & IV & & I & II & III & IV \\
\hline 1st series & 4.47 & $3(5)$ & $33(50)$ & $4(6)$ & $26(39)$ & 7.40 & $4(7)$ & $6(10)$ & $4(7)$ & $46(76)$ \\
\hline 2nd series & 6.70 & $4(50)$ & $1(12)$ & $0(0)$ & $3(38)$ & 7.40 & $2(8)$ & $6(24)$ & $0(0)$ & $17(68)$ \\
\hline 3rd series & 5.0 & $1(50)$ & $1(50)$ & $0(0)$ & $0(0)$ & 7.40 & $1(17)$ & $1(17)$ & $1(17)$ & $3(50)$ \\
\hline
\end{tabular}

Table 3. The decrease in bone marrow and kidney function by type of PRRT treatment and no. of series 
${ }^{1}$ Unit: $\times 10^{9} /$ I. Information about platelet count was registered 4 weeks after first cycle and 4 weeks after the last cycle in both series of PRRT.At baseline the platelets were available for 131 patients (98\%). For patients treated with ${ }^{90} \mathrm{Y}$, platelets-counts were registered in $37(56 \%)$ patients after the last cycle in the 1st series of PRRT. 6 (75\%) and $3(50 \%)$ were registered after the 1 st and last cycle in the 2 nd series of PRRT. 7 (58) and 5 (42\%) were registered after the $1 \mathrm{st}$ and last treatment of the 3rd series PPRT. For patients treated with ${ }^{177} \mathrm{Lu}$, platelets-counts were registered in 40 patients $(67 \%)$ and $35(58 \%)$ patients after the 1 st and last cycle of the 1st series of PRRT. $14(58 \%)$ and $10(42 \%)$ were registered after the $1 \mathrm{st}$ and last cycle of the 2nd PRRT. 3 (50\%) and 1 (17\%) were registered after the 1 st and last cycle of the 3rd series of PRRT.

2Unit: $\mathrm{ml} / \mathrm{min}$. Cr-GFR was registered before $1 \mathrm{st}$ cycle and 4 weeks after the last cycle in each series of PRRT. Cr-GFR was registered in 125 patients (94\%) at baseline. For patients treated with ${ }^{90} \mathrm{Y}, \mathrm{Cr}$-GFR was registered in 59 patients (89\%) at baseline and 51 (77\%) after 1st series of PRRT. $8(100 \%)$ and $6(75 \%)$ were registered after the 1 st and last cycle of the 2nd PRRT. 2 (100\%) and $1(50 \%)$ were registered after the $1 \mathrm{st}$ and last cycle of 3rd series of PRRT. For patients treated with ${ }^{177} \mathrm{Lu}, \mathrm{Cr}$-GFR was registered in 60 patients $(100 \%)$ at baseline and $44(73 \%)$ after 1 st series. $24(100 \%)$ and $17(71 \%)$ were registered after the 1 st and last cycle of the 2 nd. $3(50 \%)$ and $1(17 \%)$ were registered after the 1 st and last cycle of the 3rd series of PRRT.

\begin{tabular}{|l|c|c|c|c|c|c|}
\hline \multirow{2}{*}{ Type of PRRT } & \multicolumn{2}{|c|}{ 1st series of PRRT } & \multicolumn{2}{c|}{ 2nd series of PRRT } & \multicolumn{2}{c|}{ 3rd series of PRRT } \\
\cline { 2 - 7 } & \multicolumn{2}{|l|}{ From baseline to last cycle } & From 1st cycle to last cycle & From 1st cycle to last cycle \\
\hline & Platelets ${ }^{1}$ & $C r-G F R^{2}$ & Platelets & $C r-G F R$ & Platelets & $C r-G F R$ \\
\hline All & $-66(29 \%)$ & $-6(8 \%)$ & $+28(15 \%)$ & $-4(7 \%)$ & $+10(5 \%)$ & $-5(7 \%)$ \\
\hline${ }^{90} \mathbf{Y}$ & $-88(36 \%)$ & $-4(5 \%)$ & $+31(17 \%)$ & $-7(9 \%)$ & $+56(27 \%)$ & $-12(16 \%)$ \\
\hline${ }^{177} \mathbf{L u}$ & $-63(-29 \%)$ & $-3(9 \%)$ & $-37(16 \%)$ & $-7(11 \%)$ & $-12(7 \%)$ & $+3(5 \%)$ \\
\hline Combination & $-29(13 \%)$ & $-9(10 \%$ & $-64(31 \%)$ & $-4(6 \%)$ & & \\
\hline
\end{tabular}

Table 4. Anemia - Common Toxicity Criteria Scale

${ }^{*}()$ percentage of the patients who received the given isotype in the given series

At the first series the hemoglobulin were available for 40 (60\%) patients treated with ${ }^{90}$ Y, 40 (67\%) patients treated with ${ }^{177} \mathrm{Lu}$ and $7(100 \%)$ patients treated with combination. At the second series the hemoglobulin were available for 7 (88\%) patients treated with ${ }^{90} \mathrm{Y}, 14(56 \%)$ patients treated with ${ }^{177} \mathrm{Lu}$ and $2(67 \%)$ patients treated with combination. At the third series the hemoglobulin were available for 2 (100\%) patients treated with ${ }^{90} \mathrm{Y}$ and for $2(33 \%)$ patients treated with ${ }^{177} \mathrm{Lu}$. No one were treated with the combination at the 3rd series. 


\begin{tabular}{|l|c|c|c|c|c|c|c|c|c|}
\hline Grade & \multicolumn{3}{|c|}{ 1st series } & \multicolumn{3}{c|}{ 2nd series } & \multicolumn{3}{c|}{ 3rd series } \\
\hline & ${ }^{90} Y$ & ${ }^{177} \mathrm{Lu}$ & Combination & ${ }^{90} \mathrm{Y}$ & ${ }^{177} \mathrm{Lu}$ & Combination & ${ }^{90} \mathrm{Y}$ & ${ }^{177}$ Lu & Combination \\
\hline III & 0 & $2(3 \%)$ & 0 & 0 & 0 & 0 & 0 & 0 & \\
\hline IV & $1(1,5 \%)$ & $1(2 \%)$ & 0 & 0 & 0 & 0 & $1(50 \%)$ & 0 & \\
\hline
\end{tabular}

\section{Figures}

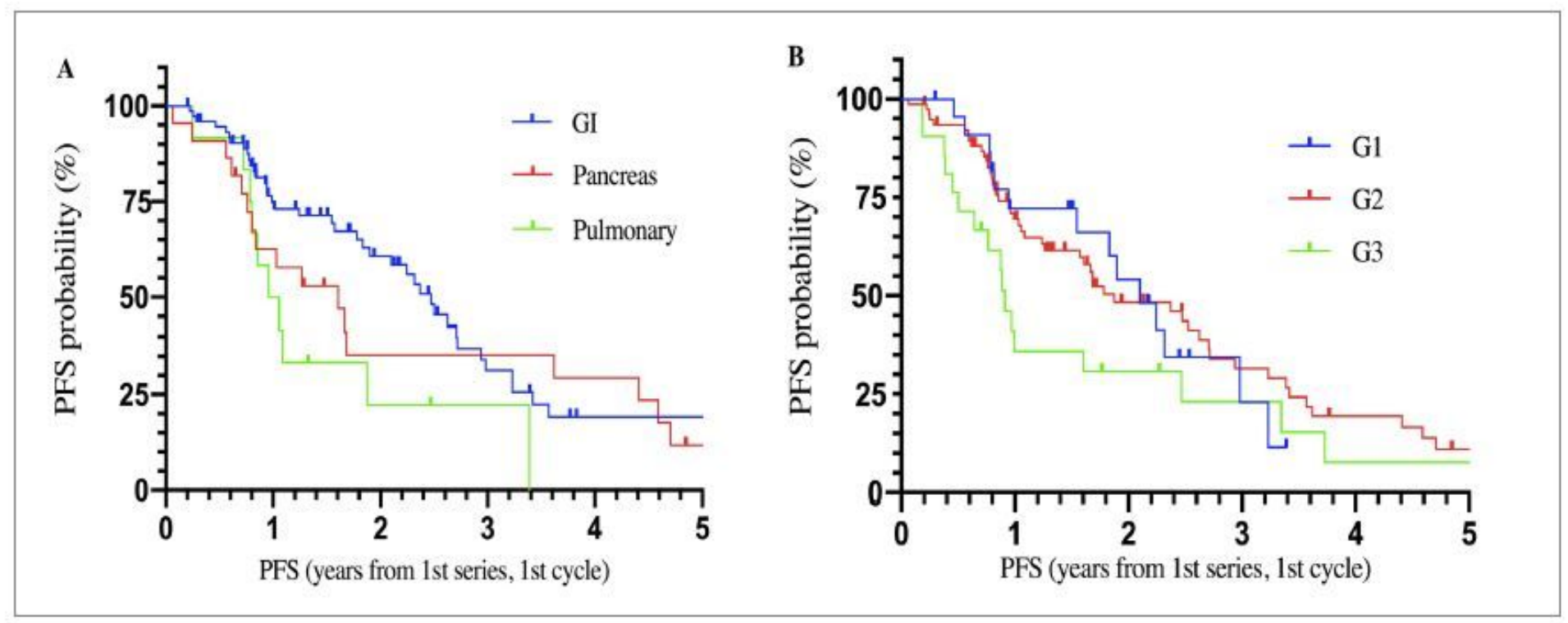

Figure 1

Progression Free Survival after first series of PRRT A PFS of all G1 and G2 tumors stratified according to primary tumor after the 1st series of PRRT. There was no significant difference between GI NET ( $n=75)$ and pancreatic NET $(n=22)$, nor between BP NET $(n=12)$ and pancreatic NET. There was a significant difference GI NET and BP NET ( $p$-value<0.05). B PFS stratified by tumor grade after the 1 st series of PRRT. G1 ( $n=23), G 2(n=77)$ and G3 (n=21). (NS).
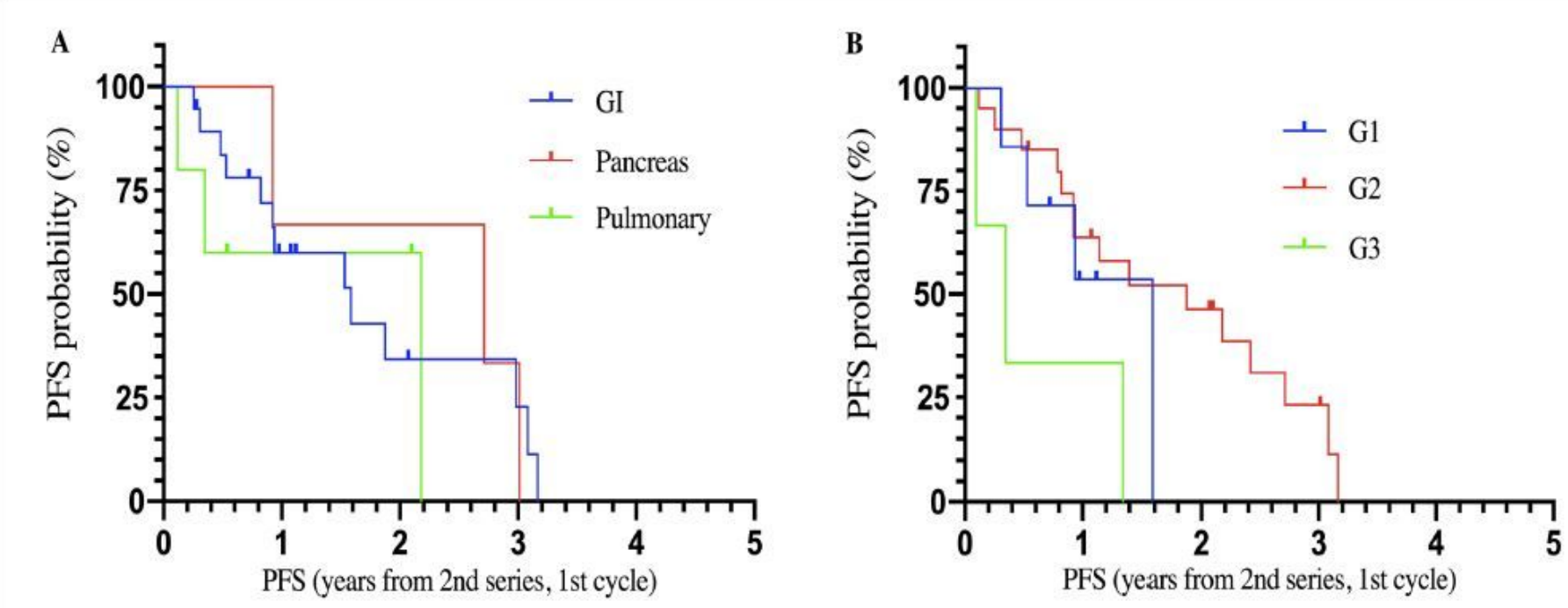
Figure 2

Progression Free Survival after second series of PRRT A PFS of all G1 and G2 tumors stratified according to primary tumor after the 2 nd series PRRT, 1 st cycle. There was no significant difference between GI NET $(n=19)$, pancreatic NET $(n=3)$ and BP NET $(n=5)$. BPFS stratified by tumor grade after the 1 st series of the 2nd PRRT series, 1 st cycle. No significant differ-ence between $G 1(n=7)$ and $G 2(n=20)$, nor between $G 1$ and G3 $(n=3)$. There was a significant difference between $G 1$ and $G 3$ ( $p$-value=0,02).

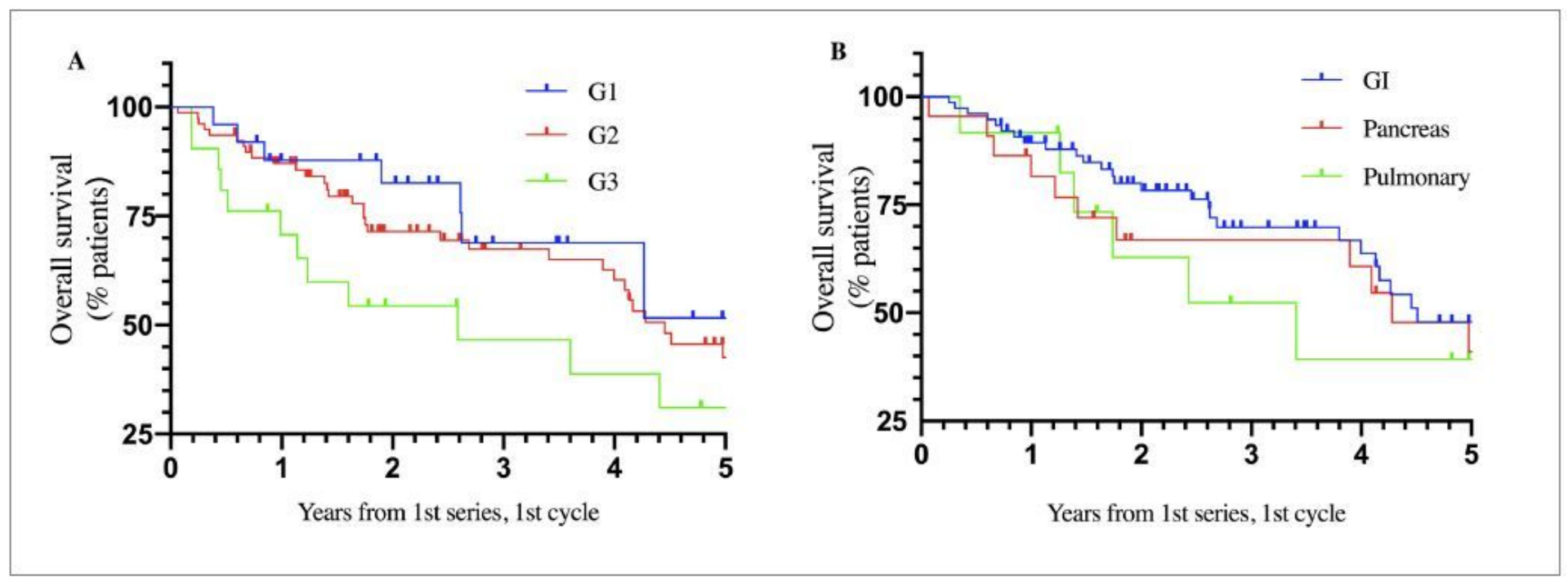

\section{Figure 3}

Overall Survival A Comparison of OS by tumor grade. There was no significant difference between G1 $(n=24), G 2(n=78)$ and $G 3(n=21)$. B Comparison of OS according to primary tumor. GI NET $(n=82)$, pancreatic NET ( $n=31)$ and pulmonary NET $(n=14)$. (G3 tumors were excluded). There was no significant difference. 